\title{
Socio-Demographic Characteristics of Substance Abuse: A Cross-Sectional Study among Male Patients of a Drug Addiction Treatment Center in Dhaka
}

\author{
Md. Mahbubur Rahman ${ }^{1}$, Yasir Arafat SM²*, Syed Emamul Hossain ${ }^{3}$, Md. Motahar Hossain ${ }^{4}$, Md Abdus Salam , \\ Quazi Lutful Kabir ${ }^{3}$
}

\author{
1Department of Psychiatry, Colonel Malek Medical College, Manikgonj, Bangladesh \\ 2Department of Psychiatry, CARe Medical College, Dhaka, Bangladesh \\ ${ }^{3}$ Central Drug Addiction Treatment Center, Tejgaon, Dhaka, Bangladesh \\ ${ }^{4}$ Department of Medicine, Colonel Malek Medical College, Manikgonj, Bangladesh \\ ${ }^{5}$ Department of Psychiatry, Kushtia Medical College, Kushtia, Bangladesh
}

\section{*Corresponding Author}

Dr. S M Yasir Arafat

\section{Article History}

Received: 05.08 .2019

Accepted: 12.08 .2019

Published: 30.08 .2019

\begin{abstract}
Introduction: Substance abuse is a major health problem in Bangladesh. We examined socio-demographic characteristics of male patients with substance use disorder in drug addiction treatment center in Dhaka city. Methods: This descriptive cross-sectional study was conducted in Central Drug Addiction Treatment Center (CTC), Tejgaon, Dhaka, Bangladesh from January 2012 to June 2013. Data were collected by administrating a semi-structured questionnaire comprised of socio demographic variables, family characteristics and substance related variables. A total of 136 male patients were interviewed purposively by face to face from outpatient department of CTC. Substance use disorder was established on the basis of DSM-IV TR by the Structured instrument SCID-CV (Structured Clinical Interview for DSM-IV Axis-1 Disorders- Clinical Version). Patients with comorbid psychiatric disorder and other organic brain disorders were not included in this study. Results: The mean age of 136 patients was $30.63 \pm 7.23$; about $42 \%$ were in $26-30$ years age group, most respondents (48.5\%) were unemployed, $95 \%$ were Muslim, and $70 \%$ urban dwellers. About $85 \%$ (115) of the patients started their using drugs by cannabis, about $9 \%$ took Phensidyl as a first drug, about $59 \%$ (80) took more than one drugs. In this study, 76 out of 136 patients took drugs by smoking which constitute $55.9 \%$, followed by injections (37.5\%). Conclusions: Cannabis was the most common as the first illicit drug used by the patients. The result suggested that 18-30-year age group was most commonly abusing time. Preventive programs could be targeted on this age group.
\end{abstract}

Keywords: Substance Dependence, Socio-demographic Characteristics, Bangladesh.

\section{INTRODUCTION}

Substance abuse is a challenging public health and social problem in Bangladesh [1]. The use of drugs has been known since ancient period. In a study, conducted in the outpatient department of National Institute of Mental Health in Dhaka, revealed that $7.66 \%$ of respondents suffered from a substance-related disorder [2]. A national survey on mental health showed that $0.63 \%$ of the adult population (18 years and above) in Bangladesh suffered from substance abuse disorder [3]. Another study conducted in a private psychiatric clinic in Dhaka showed that $29.6 \%$ of admitted psychiatric patients were suffering from substance-related disorder [4]. Illicit drug use and trafficking not only retards economic development of countries but also contribute to the spread of crimes, violence and corruption [5]. According to World Health Organization, the use of psychoactive substances causes significant health and social problems for the people who use them, and also for others in their families and communities [6].

Abuse of illicit drugs has been increasing day by day in Bangladesh, though the exact precision in unavailable [1]. The mentionable illicit drugs are opium derivatives, cannabis, stimulants (yaba, cocaine), hypnotics, cough syrup (phensidyl, dexpotent etc.) [1]. Early adult males are abusing drugs in the country [1] and heroin was the most abused drug in the country which may be changed recently [1]. However, substance abuse is under studied in Bangladesh and there is dearth of epidemiological research on it. Therefore, we aimed to see the sociodemography and pattern of substance use among the male patents in Central Drug Addiction Treatment Center of Bangladesh.

Copyright @ 2019: This is an open-access article distributed under the terms of the Creative Commons Attribution license which permits unrestricted use, distribution, and reproduction in any medium for non commercial use (NonCommercial, or CC-BY-NC) provided the original author and source are credited. 


\section{MethOdS}

This descriptive cross-sectional study was conducted in Central Drug Addiction Treatment Center (CTC), Tejgaon, Dhaka, Bangladesh from January 2012 to June 2013. Data were collected by administrating a semi-structured questionnaire comprised of socio demographic variables, family characteristics and substance related variables. Sociodemographic variables included age, marital status, educational attainment, religious practice, occupation and permanent residence area. Family characteristics include type of family, number of family members, economic status of the family, family history of substance use and earning person of the family. Substance related variables include duration of use substance use, age of substance initiation, daily expenditure for substance, type and number of substances, and route of drug administration. A total of 136 (51 injectable and 81 non-injectable substance dependence) male patients were interviewed purposively by face to face from outpatient department of CTC. Substance use disorder was established on the basis of DSM-IV TR by the Structured instrument SCID-CV (Structured Clinical Interview for DSM-IV Axis-1 Disorders- Clinical Version). Patients with comorbid psychiatric disorder and other organic brain disorders were not included in this study. After collection, data were cleaned and analyzed by Statistical Package for the Social Science, version 20 along with Microsoft Excel version 2010. Data were analyzed on the basis of the objective of the study and expressed as descriptive approach in mean, frequency and percentages.

Current study was conducted complying the declaration of Helsinki 1964. Before starting this study, the research protocol was approved by the Institutional Review Board of Bangabandhu Sheikh Mujib Medical University (BSMMU), Dhaka. Steps of the study were supervised by department of Psychiatry, BSMMU. Informed written consent was obtained from the patients without any influences. Data were collected anonymously; confidentiality of data was ensured adequately and any unauthorized access to data was not possible.

\section{RESULTS}

A total of 136 patients with substance use disorder were evaluated with using semi structured questionnaire to see their sociodemography and pattern of substance abuse. The mean $( \pm S D)$ age of patients was $30.63 \pm 7.23$ years ranging from $18-50$ years. Out of 136 patients, majority (41.9\%) were in $26-30$ years followed by $18-25$ years group $(20.6 \%)$, about $37 \%$ studied up to primary level, about $47 \%$ were married, $48.5 \%$ were unemployed (Table 1 ).

Table-1: Demographic characteristics of respondents $(n=136)$

\begin{tabular}{|c|c|c|}
\hline Variable & Frequency & Percent \\
\hline \multicolumn{3}{|l|}{ Age in years } \\
\hline $18-25$ & 28 & 21 \\
\hline $26-30$ & 57 & 42 \\
\hline $31-35$ & 23 & 17 \\
\hline $36-40$ & 13 & 9 \\
\hline $41 \&$ above & 15 & 11 \\
\hline \multicolumn{3}{|c|}{ Educational qualification } \\
\hline Illiterate & 14 & 10.3 \\
\hline Primary & 50 & 36.8 \\
\hline Secondary & 47 & 34.6 \\
\hline Higher secondary & 18 & 13.2 \\
\hline Graduate & 6 & 4.4 \\
\hline Master degree & 1 & 0.7 \\
\hline \multicolumn{3}{|l|}{ Marital status } \\
\hline Married & 64 & 47.1 \\
\hline Unmarried & 51 & 37.5 \\
\hline Divorced & 7 & 5.1 \\
\hline Others & 14 & 10.3 \\
\hline \multicolumn{3}{|l|}{ Religion } \\
\hline Islam & 130 & 95.6 \\
\hline Hindu & 6 & 4.4 \\
\hline \multicolumn{3}{|l|}{ Occupation } \\
\hline Service & 12 & 8.8 \\
\hline Business & 34 & 25 \\
\hline Student & 2 & 1.5 \\
\hline Laborer & 16 & 11.8 \\
\hline Unemployed & 66 & 48.5 \\
\hline Others & 6 & 4.4 \\
\hline \multicolumn{3}{|l|}{ Residence } \\
\hline Urban & 95 & 69.9 \\
\hline Rural & 41 & 30.1 \\
\hline Total & 136 & 100 \\
\hline
\end{tabular}


Table-2: Family characteristics of the respondents $(n=136)$

\begin{tabular}{|l|c|c|}
\hline \multicolumn{1}{|c|}{ Variables } & Grouping & $\mathbf{n}(\%)$ \\
\hline Type of family & Joint Family & $85(62.5)$ \\
\cline { 2 - 3 } & Nuclear Family & $51(37.5)$ \\
\hline \multirow{3}{*}{ Monthly family income (in BDT) } & $1-10000$ & $91(66.9)$ \\
\cline { 2 - 3 } & $10001-20000$ & $43(31.6)$ \\
\cline { 2 - 3 } & $20001-30000$ & $2(1.5)$ \\
\hline Other family members taking drugs & Yes & $13(9.6)$ \\
\cline { 2 - 3 } & No & $123(90.4)$ \\
\hline Main earning person of the family & Yes & $48(35.3)$ \\
\cline { 2 - 3 } & No & $88(64.7)$ \\
\hline
\end{tabular}

Among the 136 patients, $85(62.5 \%)$ patients lived in joint family and $51(37.5 \%)$ in nuclear family. In this study, 66.9\% patients' monthly family income was 10,000 or less followed by $31.6 \%$ were in $10,000-20,000$ income group. Out of 136 patients, only $13(9.6 \%)$ patients reported that their family member took drugs. Regarding earning capability 48 out of 136 patients said that, they were the main earning person of the family. Regarding duration of use of principal substance mean duration of use was $6.15 \pm 4.88$ in years and duration range was 06 months to 22 years. The mean age at first use of substance was $18.56 \pm 4.88$ in years and range was 10-40 years. The mean daily expenditure for substance was $377.35 \pm 257.53$ and range was $100-2000$ BDT daily (Table 2). About $85 \%$ of the patients began drug abuse with cannabis, $9 \%$ with heroin, about $59 \%$ (80) took more than one drugs, $56 \%$ took drugs by smoking, followed by injection (37.5\%) and swallowing (6.6\%) (Table 3).

Table-3: Substance use characters

\begin{tabular}{|l|c|c|}
\hline Variables & Mean $\mathbf{E S D}$ & Range \\
\hline Duration of use substance use & $6.15 \pm 4.88$ & 06 Months - 22 year \\
\hline Age at first use (years) & $18.56 \pm 4.72$ & $10-40$ years \\
\hline Daily expenditure (BDT) & $377.35 \pm 257.53$ & $100-2000$ \\
\hline Name of the initiating drug & Frequency & Percentages \\
\hline Cannabis & 116 & 85.29 \\
\hline Phensidyl & 12 & 8.82 \\
\hline Heroin & 5 & 3.68 \\
\hline Yaba (Amphetamine) & 2 & 1.47 \\
\hline Alcohol & 1 & 0.74 \\
\hline Currently taking more than one drug \\
\hline Yes & 80 & 58.80 \\
\hline No & 56 & 41.20 \\
\hline Route of drug administration & 76 & 55.90 \\
\hline Smoking & 51 & 37.50 \\
\hline Injection & 9 & 6.60 \\
\hline Oral & \multicolumn{2}{|c|}{} \\
\hline
\end{tabular}

\section{Discussion}

This study sought to identify some socio-demographic factors which may be related to patients attending to a government hospital suffering from substance abuse. The highest age range of the first substance abuse by the abusers in the present study is between 18-30 years, accounting for $70 \%$ of the population of the abusers, mean age of patients was $30.63 \pm 7.23$ ranging from 18-50 years. This finding is consistent with the findings done by Riya et al. [7] and Rahman et al. [8] which were $28.29 \pm 7.37$ and 25.3 years respectively. The Bangladesh Bureau of Statistics (2013) found that 20-34 years was the age of initiation for most (47.3\%) intoxicating substance abusers and $33 \%$ of abusers started between $15-19$ years old [9].

The study also looked at the educational levels and the dropouts in abusers. From the distribution, it is evident that the greater number of substance abusers dropped out of school at the primary school level (36.8\%) and at secondary level $34.6 \%$. In a study done by Riya et al. in different private rehabilitation centers it was found that $24 \%$ were found to have education up to primary level, $18.7 \%$ illiterate [7]. In this current study it was found that $69.9 \%$ patients were from urban and $30.1 \%$ from rural areas. In a study it was found that $78.67 \%$ from urban areas and $14 \%$ in rural areas [7]; it was found that $82.9 \%$ from urban areas in another study [10]. From above studies it reveals that drug abuse is increasing in rural areas. This study revealed $48.5 \%$ respondents were unemployed, $25 \%$ businessmen, about $9 \%$ service holder which is similar with other studies in the country such as Rahman et al found, $56.1 \%$ respondents were unemployed [8], 33.3\% respondents were unemployed in another study [7]. Current study revealed married persons were abusing drugs more than unmarried and singles which is similar with other studies in Bangladesh where $62 \%$ drug abusers were 
married [11]. About $67 \%$ respondents family income were below 10,000 taka per month which is similar with previous studies in the country such as a study done by Riya et al. found $50.2 \%$ respondents family income were below 10,000 BDT (Taka) per month.

Current study revealed about $56 \%$ patients taking drugs by inhalation, $37.5 \%$ by injection and $6.6 \%$ by swallowing; $58.8 \%$ patients took more than drug; about $85 \%$ of the patients began drug abuse with cannabis. This mode of drug taking is similar with previous studies. In a study done by Riya et al. found $65.5 \%$ patients take drug by inhalation or smoking, $30 \%$ by swallowing and $12.5 \%$ by injection [7]. Smoking or inhalation was the route used by most $(90.5 \%)$ respondents, $81.9 \%$ also used oral and $14.3 \%$ parenteral routes; about $91 \%$ abused more than 1 drug [12].

Current study has several limitations such as small sample size, single center-based study, hospital based study, purposive sampling technique, only male gender which would be considered during generalization of the study results. Despite the limitations this study provides information about socio-demographic characteristics of patients attending in government drug addiction treatment center in Dhaka.

\section{CONCLUSION}

In a summery, cannabis was the most common illicit drug abused by the patients, unemployment was an important variable which needs to be decided either as risk factor of consequence of drug abuse and 18-30 years age group was most vulnerable time to abuse drugs. Preventive programs could be targeted on this age group. Unemployed group also should be focused. We hope these findings highlight the important characteristics of drug abuse patients which could be helpful for better management.

\section{REFERENCES}

1. Kamal, M., Huq, N., Mali, B., Akter, H., Arafat, S. M. Y. (2018). Epidemiology of Substance Abuse in Bangladesh: A Narrative Review. Journal of Mental Disorders and Treatment, 4(2), 1000165.

2. Mohit, M. A., (2001). Diagnosis of patients attending outpatient department (OPD) of NIMH. Bangladesh Journal of Psychiatry, 15(1), 5-12.

3. Firoz, A. H., Karim, M. E., Alam, M. F., Rahman, A. H, Zaman, M. M. (2006). Prevalence, medical care, awareness and attitude towards mental illness in Bangladesh. Bangladesh Journal of Psychiatry, 20(1), 9-36.

4. Fahmida, A., Wahab, M. A., Rahman, M. M. (2009). Pattern of psychiatric morbidity among the patients admitted in a private psychiatric clinic. Bangladesh Journal of Medical Science, 8(1), 23-28.

5. Common wealth youth programme, Asia centre. (1991). "A peer approach, "training manual on health living without drug, 'Asia Centre, Chandigarh, India, 54-86.

6. The global burden of disease. (2004). World Health Organization, Geneva, Switzerland.

7. Riya, S., Rahman, M., Sadeque, M. Z., Kabir, A., Umar, B. U. (2013). Pattern of drug abuse among patients in some selected addiction rehabilitation centers in Dhaka city. Faridpur Medical College Journal, 8(2), 63-66.

8. Rahman, M., Zaman, M. S., Sakamoto, J., Fuikui, T. (2004). How much do drug abusers pay for drugs in Bangladesh? Journal of Health, Population and Nutrition, 22(1), 98-99.

9. Bangladesh Bureau of Statistics. (2013). Health and morbidity status survey 2012. Statistics and informatics division, Ministry of Planning, Government of the People's Republic of Bangladesh. Use of tobacco and abuse of intoxicating substances, Chapter 9 , 63-67.

10. Habib, A. K. M. (2001). Life style of drug addicts. [dissert] NIPSOM, 2000-2001:63-66.

11. Ahsan, M. N., Alam, M. F., Ahmed, S. (1999). Substance dependence: urine analysis of one hundred and forty four patients. Bangladesh Journal of Psychiatry, 13(2), 44-49.

12. Maruf, M. M., Khan, M. Z. R., Jahan, N. (2016). Pattern of Substance Use: Study in a De-addiction Clinic. Oman Medical Journal, 31(5), 327-331. 